\title{
Semi-self-pumped phase-conjugate mirrors
}

\author{
Mark Cronin-Golomb \\ Electrooptics Technology Center, Tufts University, Medford, Massachusetts 02148
}

Amnon Yariv

California Institute of Technology, Pasadena, California 91125

Received April 4, 1987; accepted June 15, 1987

\begin{abstract}
We describe semi-self-pumped photorefractive phase-conjugate mirrors based on the unidirectional ring resonator and the double phase-conjugate mirror. Their applicability to thresholding phase conjugation and one-way imaging through distortions is discussed.
\end{abstract}

In the past, four-wave mixing phase-conjugate mirrors (PCM's) have been either externally pumped or completely self-pumped. In the first case, both pumps are provided independently of the signal and must be arranged to be plane waves or at least good phase conjugates of each other. The second case is that of the passive (self-pumped) photorefractive phase-conjugate mirrors (PPCM's). For the linear $\mathrm{PPCM}^{1}$ and the cat $\mathrm{PPCM}^{2}$ both pumping beams are self-induced as oscillation beams. For the ring $\mathrm{PPCM}^{1}$ one is selfinduced and the other is provided directly by the signal. In this Letter we introduce the concept of semiself-pumped PCM's in which one pump is externally supplied to a four-wave mixing phase conjugator while the other is self-induced as an oscillation beam. One application is as a thresholding PCM, which would be useful, for example, in providing nonlinear feedback for optical associative memories. ${ }^{3-5}$ The second application is in one-way imaging through optical distortions using a phase-conjugate window ${ }^{6}$.

The first semi-self-pumped PCM (Fig. 1) is based on the unidirectional ring resonator. ${ }^{7}$ The signal beam pumps a photorefractive crystal to supply gain for unidirectional ring oscillation $I_{1}$. This unidirectional ring oscillation serves as the self-induced pump of the semi-self-pumped phase conjugation. It is forced into the lowest-order transverse mode by an intracavity spatial filter. One of the resonator cavity mirrors is partially transmitting, and the second pumping beam $I_{2}$ is injected externally through it into the ring.

The second example of a semi-self-pumped PCM is a special case of the ring semi-self-pumped PCM that results if the reflectivity of the partial-feedback mirror is reduced to zero (Fig 2). This gives us a device that was previously studied as a double PCM. ${ }^{1,8}$ Here, we discuss its interpretation as a semi-self-pumped PCM. The pump labeled 1 in the figure is the self-induced oscillation beam, while the pump labeled 2 is externally supplied.

Both of these devices may be used as thresholding PCM's finding application, for example, in optical associative memories. Associative memory devices op- erate by using nonlinear feedback to select the stored memory that most closely resembles the partial information input. The parallelism of optical information processing has made all-optical associative memories an attractive prospect, and some of the first theoretical and experimental considerations along these lines have involved phase-conjugate resonators in which the memories are stored in intracavity holograms. Memory selection takes place through competitive mode selection biased by injection of a beam bearing the partial information. ${ }^{3-5}$ The competition is encouraged by the presence of a positive thresholding effect in the reflectivities he cavity PCM's. ${ }^{9,10}$

In the semi-self-pu .nped phase conjugator of Fig. 1 the presence of the externally supplied pumping beam $I_{2}{ }^{\prime}$ suppresses buildup of the self-induced pumping beam $I_{1}$ until the signal reaches a certain threshold intensity. This threshold intensity is proportional to the intensity of the external pump. We are currently developing a full theoretical treatment of this device, which, because of the closed nature of the ring resonator, will require the simultaneous fitting of phase and

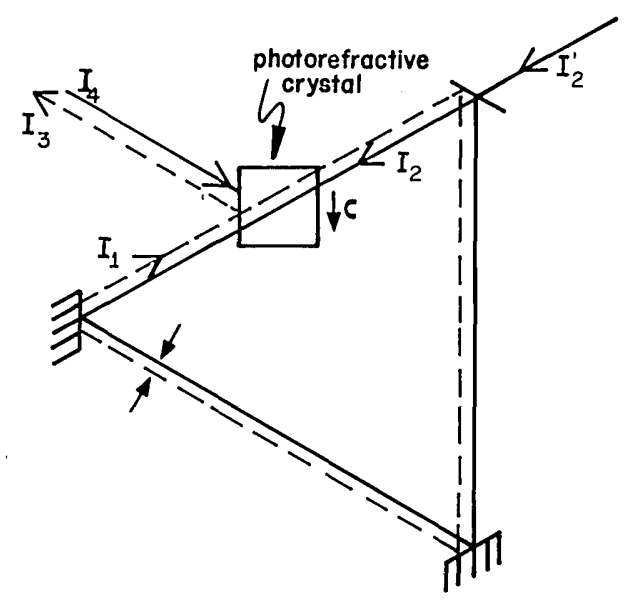

Fig. 1. Semi-self-pumped photorefractive phase conjugator based on unidirectional ring resonator. The intracavity aperture forces single-transverse-mode operation. 


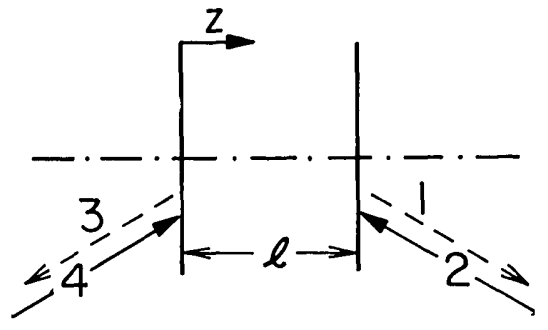

Fig. 2. The double PCM may be interpreted as a semi-selfpumped phase conjugator. When barium titanate is used, the crystal is tilted so that the $c$ axis is neither parallel nor perpendicular to the bisectors of the beams. This enables extraordinarily polarized beams to use the high electro-optic coefficient $r_{42}$.

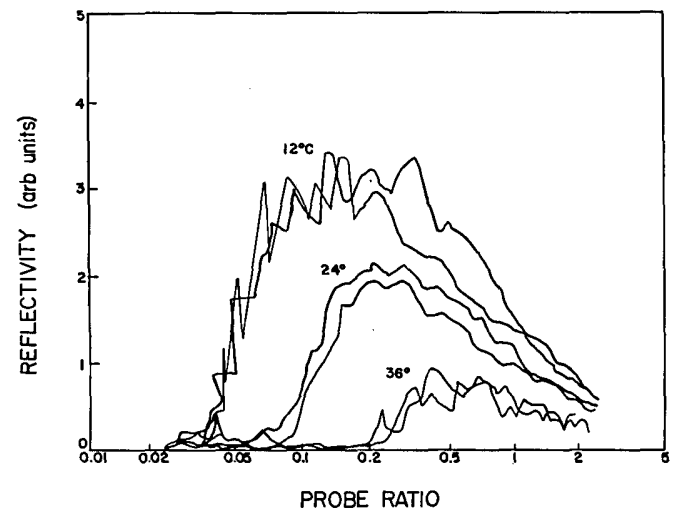

Fig. 3. Reflectivity of semi-self-pumped phase conjugator versus probe ratio for three different crystal temperatures.

amplitude boundary conditions giving rise to cavitylength-dependent oscillation frequency-detuning effects. Figure 3 shows the experimental performance of this device, giving the reflectivity of a ring-resonator barium titanate semi-self-pumped phase conjugator as a function of probe ratio, defined as signal intensity normalized by the external pump intensity. The coupling strength in barium titanate is highly temperature sensitive and increases substantially near its tetragonal to orthorhombic phase transition near $9^{\circ} \mathrm{C}$, so that temperature tuning of the device is possible, as shown by the results for three different temperatures.

The transfer function given by this device is a sigmoid thresholding function. Such functions have particular importance in associative processing ${ }^{11}$ and are characterized by being zero for arguments below threshold and then rising through an inflection toward saturation at high-valued arguments.

The double PCM, although it is structurally simpler and easier to analyze theoretically, ${ }^{1,8}$ does not have this sigmoid thresholding function. Here, there is thresholding at both high and low intensities ${ }^{8}$ because the reflectivity is nonzero only for input beam intensity ratio $q=I_{4}(0) / I_{2}(l)$ (see Fig. 2) in the range

$$
\frac{(1-a)}{(1+a)}<q<\frac{(1+a)}{(1-a)}
$$

where $a$ is given by the solution of the equation tanh $(\gamma l a / 2)+a=0, \gamma$ is the photorefractive coupling constant, and $l$ is the length of the interaction region. The theory of the double PCM gives its reflectivity $R$ as

$$
R=\left(\frac{a+1}{q}+a-1\right)\left(\frac{a-1}{q}+a+1\right) / 4,
$$

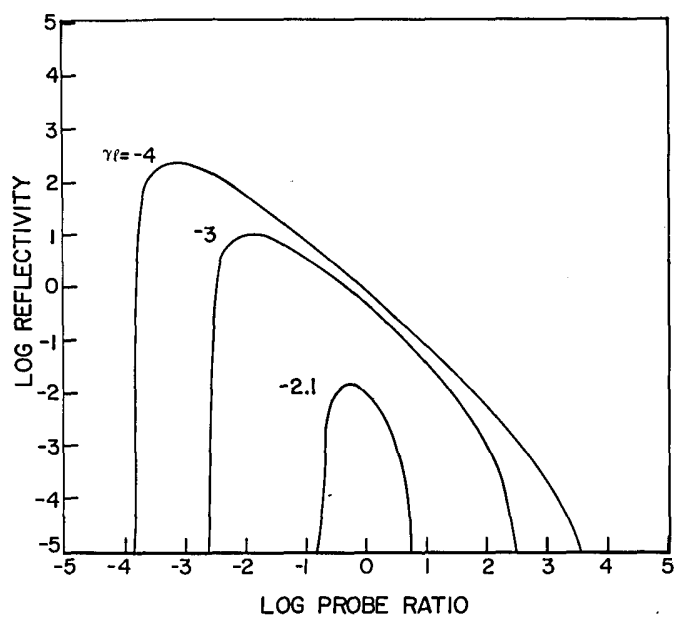

Fig. 4. Theoretical reflectivity of double phase conjugator versus probe ratio for various coupling strengths.

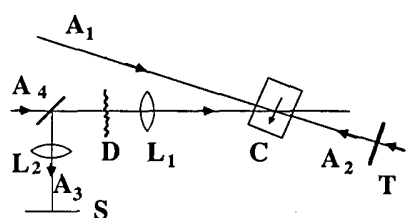

(a)

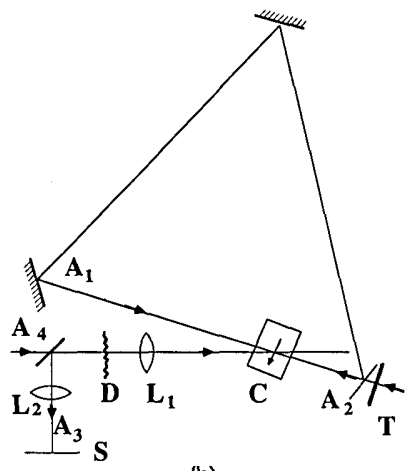

(b)

Fig. 5. (a) Original realization of phase-conjugate window with externally supplied pump. Beam $\mathrm{A}_{2}$ carries the picture information T. Probe beam $\mathrm{A}_{4}$ passes through distortion $\mathrm{D}$, which must be imaged onto the crystal by lens $L_{1}$. The reflected beam $\mathrm{A}_{3}$ carries the information through the distortion, is split off at beam splitter $\mathrm{S}$, and is imaged onto the film by lens $\mathrm{L}_{2}$. (b) Semi-self-pumped phase conjugator applied in realization of the self-pumped phase-conjugate window. The experimental results of Fig. 6 were obtained with a barium titanate crystal pumped at $514.5 \mathrm{~nm}$ by a single-longitudinal-mode argon laser. The transparencyinformation-bearing beam was frequency shifted $40 \mathrm{MHz}$ by an acousto-optic modulator to prevent the buildup of reflection gratings in the photorefractive crystal. 


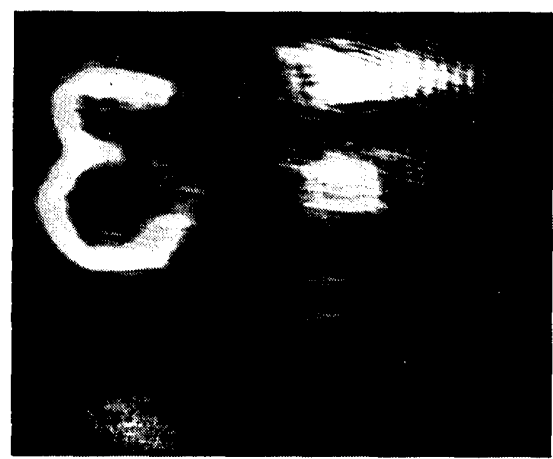

(a)

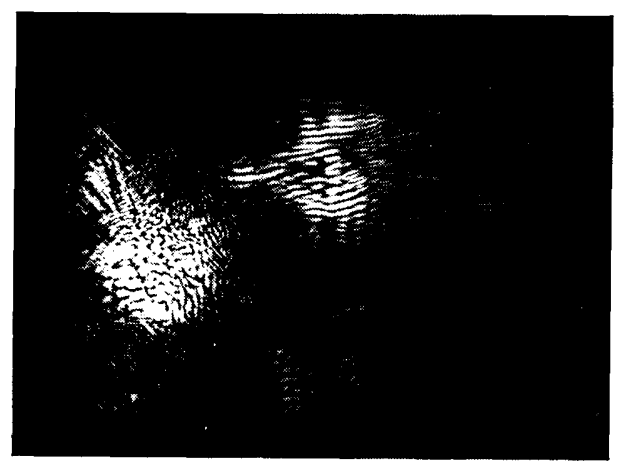

(b)

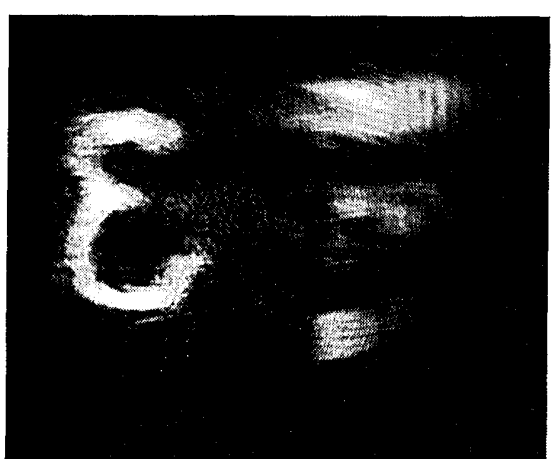

(c)

Fig. 6. (a) Transparency $\mathrm{T}$ imaged through self-pumped phase-conjugate window without distortion in the path. (b) Distortion alone by an equivalent non-phase-conjugating optical system. (c) Self-pumped phase-conjugate window with distortion in the path.

plotted in Fig. 4 as a function of the probe ratio for several values of coupling strength. That these results are similar to the experimental results for the ring semi-self-pumped PCM is understandable, given the devices' close relationship to each other.

We now turn to the application of semi-self-pumped phase conjugators to the phase-conjugate window 6 for one-way imaging through thin distortions. The original realization of the phase-conjugate window [Fig. 5(a)] was similar to an externally pumped PCM and had the disadvantage of requiring a clean pump beam coherent with the distorted probe. This meant that the clean beam had either to bypass the distortion or to be spatially filtered after passing through the distortion, thereby losing most of its intensity. In either case, the practical utility of the device was compromised. If the externally pumped PCM portion of the device is replaced with a semi-self-pumped PCM [Fig. $5(\mathrm{~b})$ ], the clean beam can be self-induced as an oscillation beam. Figure 6(a) shows an image of part of group 1 (2.52 line pairs $/ \mathrm{mm}$ ) of a U.S. Air Force resolution chart when viewed through the phase-conjugate window without any distortion in the line of sight. This gives a baseline reading of the general quality of the imaging system, which could be improved with further engineering. Figure $6(\mathrm{~b})$ is an image of the same scene when viewed through a distortion. Figure 6(c) shows the same distorted scene when corrected by the self-pumped phase conjugate window.

Finally, we note that the double phase conjugator is unsuitable for this application. Referring to Fig. 2, we have beam 1 generated as the phase conjugate of input beam 2 and beam 3 generated as the phase conjugate of input beam 4 independently of any spatial information on beam 2. To be useful as a one-way imaging device, however, beam 3 would be required to pick up the transparency information on beam 2 . The ring semi-self-pumped PCM works as a one-way imager because beam 1 is forced, by the closed oscillation cavity with intracavity aperture, to have a fixed spatial profile independent of the spatial profile of beam 2 .

In summary, we have introduced the concept of a new class of PCM, joining the already established groups of fully externally and fully self-pumped phase conjugators. Two applications of these devices have been described: positive thresholding phase conjugation for optical associative memory and one-way imaging through thin distortions.

This research was supported by the U.S. Army Research Office, Durham, North Carolina.

\section{References}

1. M. Cronin-Golomb, B. Fischer, J. O. White, and A. Yariv, IEEE J. Quantum Electron. 20, 12 (1984).

2. J. Feinberg, Opt. Lett. 7, 486 (1982).

3. D. Z. Anderson, Opt. Lett. 11, 56 (1986).

4. B. H. Soffer, G. J. Dunning, Y. Owchenko, and E. Marom, Opt. Lett. 11, 118 (1986).

5. A. Yariv and S. K. Kwong, Opt. Lett. 11, 186 (1986).

6. B. Fischer, M. Cronin-Golomb, J. O. White, and A. Yariv, Appl. Phys. Lett. 41, 141 (1982).

7. J. O. White, M. Cronin-Golomb, B. Fischer, and A. Yariv, Appl. Phys. Lett. 40, 450 (1982).

8. S. Weiss, S. Sternklar, and B. Fischer, Opt. Lett. 11, 114 (1987).

9. M. B. Klein, G. J. Dunning, G. C. Valley, R. C. Lind, and T. R. O'Meara, in Digest of Conference on Lasers and Electro-Optics (Optical Society of America, Washington, D.C., 1985), paper PD5. We note that the device described in this paper is a negative thresholder: higher inputs give lower outputs.

10. M. Cronin-Golomb and A. Yariv, Proc. Soc. Photo-Opt. Instrum. Eng. 700, 301 (1986).

11. M. A. Cohen and S. Grossberg, IEEE Trans. Syst. Man Cybern. SMC-13, 815 (1983). 